\title{
Titanium Scaffolds - Hopes and Limitations
}

\author{
Magda Dziaduszewska and Andrzej Zielinski* \\ Department of Materials Engineering and Bonding, Poland
}

*Corresponding author: Andrzej Zielinski, Department of Materials Engineering and Bonding, Gdansk University of Technology, Narutowicza 11/12, 80-233 Gdansk, Poland.

To Cite This Article: Andrzej Zielinski. Titanium Scaffolds - Hopes and Limitations. Am J Biomed Sci \& Res. 2018 - 4(6). AJBSR.MS.ID.000841. DOI: 10.34297/AJBSR.2019.04.000841

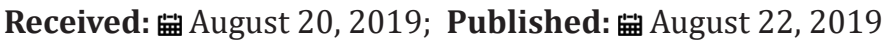

\begin{abstract}
The state-of-art on titanium scaffolds is briefly reviewed. The real hopes from medicine associated with scaffolds are indicated. The limitations of different scaffolds associated with their mechanical, chemical and biological properties are discussed. The great importance of titanium scaffolds is demonstrated as the materials of high biocompatibility and corrosion resistance, fair mechanical properties and important biological behaviour.
\end{abstract}

Keywords: Titanium; Scaffolds; Mechanical Properties; Biological Properties

\section{Brief Review}

Large bone defects caused by traumas, tumors, and/or infections [1] which size exceeds critical value of $20 \mathrm{~mm}$ do not have the ability to self-regenerate [2]. In clinical conditions, bone substitutes are used to heal such defects. Three-dimensional porous structure that provides support and allows cells to adhere, proliferate and differentiate to restore the functionality of bone is called scaffold. The reconstruction of hard tissue seems to be a big challenge, since both mechanical conditions and biological requirements comprising a number of physical and chemical properties of the biomaterial must be taken into account. The material, especially for load bearing applications, is exposed into risk such as long-term mechanical stress, bone microtraumas around the implant, inflammation, and friction of material and bone with variable amplitude leading to destruction of the bone-implant bonding [3]. Thus, the material should be characterized by the excellent properties including biocompatibility, a lack of cytotoxicity, corrosion resistance in body environment, high fatigue and wear resistance, ductility, as well as it has to demonstrate an excellent combination of high strength and low modulus [1,4]. The widely used materials for bone scaffolds are: metals (e.g. titanium alloys), ceramics (e.g. hydroxyapatite) and polymers (e.g. PLA, PCL) [4,5]. Polymers, despite their great ability of controllable biodegradability and osteo conductivity, have the mechanical resistance not fair enough. On the other hand, ceramics are characterized by better mechanical strength, however they are sensitive to brittle fracture [6]. Over the years, researchers have looked for the optimal solutions taking into consideration metals, particularly titanium alloys as a good alternative for load bearing applications. This short article briefly presents the hopes and limitations concerning the titanium scaffolds, based on recent research.

From a mechanical perspective, the most critical properties to be matched by the scaffold are bone loading stiffness, strength and fatigue strength. Implants with an appropriate elastic modulus, mimicking that of natural bone, can prevent stress-shielding [7]. Although titanium alloy modulus ranging from 110 (pure Ti) to $55 \mathrm{GPa}$ (Ti35Nb7Zr5Ta) is much smaller than conventional alloys such as stainless steel (210 GPa) and chromium cobalt alloys (240 GPa), this value is still higher than that of natural bone (4 to $30 \mathrm{GPa}$ depending on the type of the bone and the direction of measurement) [5]. Titanium and its alloys possess an excellent biocompatibility, corrosion resistance, high strength-to-weight ratio, but their wear resistance under load conditions is very limited and tends to wear heavily [4].

Considering biocompatibility in terms of cell attachment and proliferation as well as lack of toxicity and inflammatory reactions, it may be said that titanium and its alloys are well tolerated and are almost inert materials in the environment of the human body. Nevertheless, recent literature reports indicated undesirable side effects originating from an accumulation of some toxic elements causing allergic reactions (i.e. $\mathrm{Al}$ or $\mathrm{V}$ ) [8]. What is more, some researchers pointed out the necessity of improvement the osteo conductivity of titanium due to improvement of its osseointegration. One more limitation of titanium is the lack of its biodegradable property, causing that scaffold would not be a preferable temporary 
solution. However, the performance of titanium and its alloys can be significantly increased by developing the appropriate architecture both alloy and scaffold, as well as by their surface treatment.

A wide spectrum of microstructures is possible to fabricate, depending on alloys' chemistry and thermomechanical treatment. Hence, the titanium alloys seem very susceptible to customizing its properties according to specific requirements. The confirmation might be the development of low-alloy beta-titanium alloys that consist of compatible elements and have a proximal bone module [8]. In addition, theoretical research has shown that $\mathrm{Nb}, \mathrm{Zr}$, Mo and Ta are the most suitable alloying elements that can be added to reduce the modulus of elasticity without losing strength. Zirconium has the best mechanical properties and high resistance to crack propagation [9].

Recently, a breakthrough and a milestone in improving titanium alloys occurred by of manufacturing the metal scaffolds by controlled process of additive techniques (e.g. selective laser melting, electron beam melting) [10]. Those techniques show several advantages over conventional ones, including the ability to create arbitrarily complex 3D structures with highly accurate and predictable architecture. The studies have shown that the relative volume fraction of the porous structure, the degree of porosity, the size of the pores and their shape affect the mechanical properties of the implant, and along with the method and parameters of the surface modification process - also the in vitro biological properties [11]. Hence, already mentioned problem with mechanical properties and osseointegration of titanium alloys may be solved. Introducing controlled porosity to solid material makes it possible to obtain the mechanical properties similar to those of the host tissue (Young`s modulus), provides a pathway for cell growth in a porous implant, support nutrients and waste transfer as well as improves angiogenesis [12].

Equally important for the success of the loading-bearing scaffolds is the surface treatment procedure which can lead to increase of the titanium wear resistance, as well as improvement of osseointegration. Surface chemistry, surface roughness and surface topography play a major role in the development of good osseointegration, additionally coatings and specific growth factors provide the necessary bioactivity [13]. Many methods are known for both, surface treatment [14] and coating implementation [15], but in most cases they involve rather rough than porous structure. The big challenge is to determine the methodology of deposition of the coating inside the pores as well as the methodology of examination of the results.

Developing the proper microstructure with optimal mechanical properties is a difficult problem in the field of titanium alloys.
Although the titanium-based scaffolds are considered to have limited prospect in clinical applications, it can be noticed that research progress systematically goes towards elimination/ reduction of their weaknesses. It seems that a big potential of titanium in application for load-bearing scaffolds is worth the researcher's affordances.

\section{References}

1. Zhang L, Yang G, Johnson BN, Jia X (2019) Three-dimensional (3D) printed scaffold and material selection for bone repair. Acta Biomater 84: 1633.

2. Lopes D, Martins-Cruz C, Oliveira MB, Mano JF (2018) Bone physiology as inspiration for tissue regenerative therapies. Biomaterials 185: 240275 .

3. Barbas A, Bonnet AS, Lipinski P, Pesci R, Dubois G (2012) Development and mechanical characterization of porous titanium bone substitutes. J Mech Behav Biomed Mater 9: 34-44.

4. Deb P, Deoghare AB, Borah A, Barua E, Das Lal S (2018) Scaffold Development Using Biomaterials: A Review Mater Today Proc 5(5): 1290912919.

5. Wu S, Liu X, Yeung KWK, Liu C, Yang X (2014) Biomimetic porous scaffolds for bone tissue engineering. Mater Sci Eng R Reports 80(1): 1-36

6. Fahmy MD, Jazayeri HE, Razavi M, Masri R, Tayebi L (2016) Three-Dimensional Bioprinting Materials with Potential Application in Preprosthetic Surgery J Prosthodont 25(4): 310-318.

7. Yuan L, Ding S, Wen C (2019) Additive manufacturing technology for porous metal implant applications and triple minimal surface structures: A review, Bioact Mater 4(1): 56-70.

8. Chen Q, Thouas GA (2015) Metallic implant biomaterials. Mater Sci Eng R 87: 1-57.

9. Li N et al. (2019) Progress in additive manufacturing on new materials: A review. J Mater Sci Technol 35(2): 242-269.

10. Melancon D (2017) Mechanical characterization of structurally porous biomaterials built via additive manufacturing: experiments, predictive models, and design maps for load-bearing bone replacement implants. Acta Biomater 63: 350-368.

11. Zhao S, Li SJ, Hou WT, Hao L, Yang R, et al. (2016) The influence of cell morphology on the compressive fatigue behavior of Ti-6Al-4V meshes fabricated by electron beam melting. J Mech Behav Biomed Mater 59: 251-264.

12. Wang Z (2017) Analysis of factors influencing bone ingrowth into three-dimensional printed porous metal scaffolds: A review. J Alloys Compd 717: 271-285.

13. Ghassemi T et al. (2018). Current Concepts in Scaffolding for Bone Tissue Engineering. Arch Bone Jt Surg 6(2): 90-99.

14. Manjaiah M, Laubscher RF (2017) A Review of The Surface Modifications of Titanium Alloys for Biomedical Applications. Mater Techn 51(2): 181-193.

15. Ma T, Ge X, Zhang Y, Lin Y (2016) Effect of Titanium Surface Modifications of Dental Implants on Rapid Osseointegration. In: Sasaki K, Suzuki O, Takahashi N (eds.) Interface Oral Health Science Springer, Singapore 247-256. 